\title{
Comment on "Diagnostic Laparoscopy as a Selection Tool for Patients with Colorectal Peritoneal Metastases to Prevent a Non-therapeutic Laparotomy During Cytoreductive Surgery"
}

\author{
Colette R. Pameijer, MD \\ Department of Surgery, College of Medicine, The Pennsylvania State University, Hershey, PA
}

Non-therapeutic laparotomy exposes patients to unnecessary risk and is too common in the setting of colorectal peritoneal metastases. The limits of cross-sectional imaging are well known to peritoneal surgeons, and better modalities are needed to diagnose and assess peritoneal disease for treatment planning, be it cytoreductive surgery (CRS) and heated intraperitoneal chemotherapy (HIPEC) or systemic chemotherapy. Hentzen et al. ${ }^{1}$ review their experience with diagnostic laparoscopy (DLS) as a selection tool for CRS during two periods in their practice. From 2006 to 2011 patients referred to their center for CRS had an exploratory laparotomy and hence constitute the historic control group (or cohort A). DLS was introduced in 2012 as a standard in the preoperative evaluation for CRS, and patients referred between 2012 and 2019 are included in cohort B. All patients were first assessed with a computed tomography (CT) scan of the chest, abdomen, and pelvis, and only those patients deemed to be candidates for CRS had DLS. The rate of non-therapeutic laparotomy was $35.4 \%$ prior to the introduction of DLS, and decreased significantly to $21 \%$ thereafter, although not all patients in cohort B actually had DLS $(68.5 \%)$. In patients who had DLS, the rate of non-therapeutic laparotomy was only $17.6 \%$. While this represents a significant improvement, about $20 \%$ of patients still undergo a non-therapeutic laparotomy. The most common reasons to abandon surgery were a peritoneal cancer index $(\mathrm{PCI})>20$ and extent of

(C) Society of Surgical Oncology 2020

First Received: 23 October 2019;

Published Online: 14 January 2020

C. R. Pameijer, MD

e-mail: cpameijer@pennstatehealth.psu.edu small bowel involvement. This is consistent with published experience with laparoscopy, ${ }^{2,3}$ which finds that DLS underestimates PCI, although the pelvis and right hemidiaphragm are most commonly underestimated. Visibility of the abdomen during DLS was good for most patients $(74 \%)$ and the complication rate from DLS was low (3.5\%). The authors do not indicate if poor visibility was correlated with non-therapeutic laparotomy. While the selection criteria for DLS was candidacy for CRS with no exclusions for prior surgery, in cohort B the most common reason for not having DLS (66.6\%) was recent laparotomy or laparoscopy, such that DLS was redundant.

The authors suggest that DLS may be less important in the future as imaging detection is improving. Some centers prefer magnetic resonance imaging (MRI) to determine the extent of disease, and high detection rates are described, ${ }^{4}$ but this is center- and radiologist-dependent, and MRI has not been widely adopted. As imaging remains variable and unsatisfactory, rates of DLS should probably increase in this patient population. Many HIPEC centers, including our own, now routinely perform DLS, not just to document the extent of disease but also to screen patients at high risk for peritoneal recurrence. The added value of DLS is the subject of the COLOPEC II trial ${ }^{5}$ and can be inferred from the high rate of recurrent disease identified in the PROPHYLOCHIP trial, ${ }^{6}$ in which $52 \%$ of patients had peritoneal disease at the time of second-look surgery. Concerns regarding the safety of DLS in this patient population, given the high rate of prior surgery and the potential risk of port-site metastases, have not been confirmed. Hentzen et al. ${ }^{1}$ as well as prior reports, ${ }^{2,3,7}$ find no significant complications; therefore, avoiding the alternative of a non-therapeutic laparotomy is well worth the risk of a DLS. 
In this study, the median survival of patients who had CRS/HIPEC was 36.4 months. ${ }^{1}$ This prolonged survival is similar to the 41-month median survival of patients in the PRODIGE 7 trial reported at ASCO. ${ }^{8}$ In both studies, patients are treated with a combination of systemic chemotherapy and CRS/HIPEC. While there was no overall survival benefit to the addition of $30 \mathrm{~min}$ of hyperthermia with oxaliplatin, the PRODIGE 7 results demonstrate prolonged survival with surgical management of carcinomatosis. These results should be compared with the median survival of 3.6 years after resection of liver metastases, ${ }^{9}$ and a median survival of 12.7 months with systemic chemotherapy alone for peritoneal disease. ${ }^{10}$ The relevant trial questions today include selection criteria for CRS/HIPEC, sequencing of systemic therapy and surgery, and determining the role of intraperitoneal agents. The question of systemic therapy versus CRS/HIPEC does not align with current practice patterns and is unlikely to accrue patients. ${ }^{11,12}$

Hentzen et al. ${ }^{1}$ describe several important trends in their study. The patients in cohort B were older and more often presented with metachronous disease, and only $18.6 \%$ of patients had a PCI $>20$, compared with $45.5 \%$ in cohort A. Taken together, this reflects a trend of increasing acceptance of CRS/HIPEC as a treatment option for colorectal peritoneal metastases in The Netherlands, and probably better patient selection preoperatively. This parallels the trend in the US, where there are now over 100 centers that offer HIPEC and over 1500 cases performed annually (Ellis, SSO Regional Therapy Meeting 2019). What is not paralleled in the US is the clinical trial experience. The Europeans have completed PRODIGE 7, PROPHYLOCHIP, and COLOPEC, and COLOPEC II is now open. HIPEC trials for ovarian cancer have also been recently completed in The Netherlands ${ }^{13}$ and are open in Spain (NCT02681432) and France (NCT03842982). The Eastern Cooperative Oncology group now has a HIPEC Working Group, but it remains difficult to get trials through the hierarchy of committees. The enthusiasm for HIPEC trials in the US is dampened by the poor accrual of ACOSOG Z6091, which intended to randomize patients with colon cancer and peritoneal metastases to systemic chemotherapy versus CRS/HIPEC. This design was not acceptable to patients or HIPEC surgeons as potential surgical candidates could be randomized to systemic therapy alone. The only prospective and multi-institutional HIPEC trial completed in the US is the trial of oxaliplatin versus mitomycin $\mathrm{C}$ in patients with appendiceal cancer ${ }^{14}$ led by Dr. Levine at Wake Forest. Nonetheless, the tide is rising, and the upcoming publication of the Chicago Consensus guidelines ${ }^{15}$ reflects a level of coordination of HIPEC centers not previously seen in the US. With the current number of HIPEC centers and invested surgeons, coupled with better trial design, the US should be able to open and complete clinical trials for patients with colorectal peritoneal metastases.

DISCLOSURE Colette R. Pameijer declares no conflicts of interest.

\section{REFERENCES}

1. Hentzen J, Constansia RDN, Been LB, Hoogwater FJH, van Ginkel RJ, van Dam GM, et al. Diagnostic laparoscopy as selection tool for patients with colorectal peritoneal metastases to prevent a non-therapeutic laparotomy during cytoreductive surgery. Ann Surg Oncol. 2019. https://doi.org/10.1245/s10434-01907957-w.

2. Jayakrishnan TT, Zacharias AJ, Sharma A, Pappas SG, Gamblin TC, Turaga KK. Role of laparoscopy in patients with peritoneal metastases considered for cytoreductive surgery and hyperthermic intraperitoneal chemotherapy (HIPEC). World J Surg Oncol. 2014; 12:270.

3. Tabrizian P, Jayakrishnan TT, Zacharias A, et al. Incorporation of diagnostic laparoscopy in the management algorithm for patients with peritoneal metastases: a multi-institutional analysis. Journal of surgical oncology. 2015;111(8):1035-40.

4. Low RN, Barone RM, Lee MJ. Surveillance MR imaging is superior to serum tumor markers for detecting early tumor recurrence in patients with appendiceal cancer treated with surgical cytoreduction and HIPEC. Ann Surg Oncol. 2013;20(4):1074-81.

5. Bastiaenen VP, Klaver CEL, Kok NFM, et al. Second and third look laparoscopy in pT4 colon cancer patients for early detection of peritoneal metastases; the COLOPEC 2 randomized multicentre trial. BMC Cancer. 2019;19(1):254.

6. Goere D, Glehen O, Quenet F, et al. Results of a randomized phase 3 study evaluating the potential benefit of a second-look surgery plus HIPEC in patients at high risk of developing colorectal peritoneal metastases (PROPHYLOCHIPNTC01226394). J Clin Oncol. 2018;36(15 Suppl):3531.

7. Iversen LH, Rasmussen PC, Laurberg S. Value of laparoscopy before cytoreductive surgery and hyperthermic intraperitoneal chemotherapy for peritoneal carcinomatosis. The British journal of surgery. 2013;100(2):285-92.

8. Quenet F, Elias D, Roca L, et al. A UNICANCER phase III trial of hyperthermic intra-peritoneal chemotherapy (HIPEC) for colorectal peritoneal carcinomatosis (PC): PRODIGE 7. J Clin Oncol. 2018;36(18 Suppl):LBA3503.

9. Kanas GP, Taylor A, Primrose JN, et al. Survival after liver resection in metastatic colorectal cancer: review and meta-analysis of prognostic factors. Clin Epidemiol. 2012;4:283-301.

10. Franko J, Shi Q, Goldman CD, et al. Treatment of colorectal peritoneal carcinomatosis with systemic chemotherapy: a pooled analysis of north central cancer treatment group phase III trials N9741 and N9841. J Clin Oncol. 2012;30(3):263-7.

11. Elias D, Delperro JR, Sideris L, et al. Treatment of peritoneal carcinomatosis from colorectal cancer: impact of complete cytoreductive surgery and difficulties in conducting randomized trials. Ann Surg Oncol. 2004;11(5):518-21.

12. Cashin PH, Mahteme H, Spang N, et al. Cytoreductive surgery and intraperitoneal chemotherapy versus systemic chemotherapy for colorectal peritoneal metastases: a randomised trial. Eur $J$ Cancer. 2016;53:155-62.

13. van Driel WJ, Koole SN, Sikorska K, et al. Hyperthermic Intraperitoneal chemotherapy in ovarian cancer. $N$ Engl $\mathrm{J}$ Med. 2018;378(3):230-40. 
14. Levine EA, Votanopoulos KI, Shen P, et al. A multicenter randomized trial to evaluate hematologic toxicities after hyperthermic intraperitoneal chemotherapy with oxaliplatin or mitomycin in patients with appendiceal tumors. J Am Coll Surg. 2018;226(4):434-43.

15. Chicago Consensus Working Group. Chicago Consensus on peritoneal surface malignancies. Ann Surg Oncol (In press).
Publisher's Note Springer Nature remains neutral with regard to jurisdictional claims in published maps and institutional affiliations. 